\title{
¿Cómo acercarse a historias traumáticas en contextos de rehabilitación psicosocial? Un estudio de caso de un paciente de esquizofrenia paranoide
}

\author{
How can we approach to traumatic stories \\ in psychosocial rehabilitation contexts? \\ A case study of a patient of paranoid schizophrenia
}

Javier Saavedra Macías ${ }^{1}$

${ }^{1}$ Licenciado en Psicología. Psicoanalista. Diplomado en Salud Mental en Situaciones de Violencia Política y Catástrofes. Docente-Investigador del Programa Nacional de Formación del Profesorado Universitario, Departamento de Psicología Experimental, Universidad de Sevilla, España. fjsaavedra@us.es
RESUMEN Las historias que construimos sobre nuestras vidas han sido postuladas como uno de los instrumentos esenciales para la construcción de la identidad social. Sin embargo, la psicología y las ciencias sociales han prestado poca atención a la elaboración de narrativas de vida de pacientes esquizofrénicos en proceso de rehabilitación, especialmente a los pacientes que viven en residencias o "casas hogares" específicas de carácter social. El presente artículo describe una experiencia de acercamiento a una historia personal de carácter traumático de un paciente de esquizofrenia paranoide en el marco de un centro residencial de carácter social. Se exponen los fundamentos teóricos de la psicología narrativa y se defiende la necesidad de una narrativa de vida coherente y compartible para la completa recuperación del paciente. Se discute la necesidad de que los centros de carácter social de atención a personas con trastornos mentales graves, apoyen la construcción de narrativas de vida coherentes. El autor propone el término de "acompañamiento narrativo" para referirse a los procesos de reelaboración de la historia personal en contextos no clínicos.

PALABRAS CLAVE Narrativas Personales; Esquizofrenia; Rehabilitación; Acontecimientos que Cambian la Vida; Servicios Comunitarios de Salud Mental.

\begin{abstract}
The stories, which we construct about our own lives, have been postulated as one of the essential instruments for the construction of the social identity. Nevertheless, Psychology and Social Sciences have paid little attention to the elaboration of life narratives of schizophrenic patients in rehabilitation process, especially patients who live in residences or "supported houses" of a specific social character. This article describes an experience of approach to a personal traumatic story which belongs to a patient of paranoid schizophrenia in the setting of a social residential center. The theoretical essentials elements of the narrative psychology are showed in the present work and it's supported the need for a coherent and shareable life narrative in order to achieve completely the patients' recovery. Moreover, the necessity is discussed that supported houses assisting people with serious mental disorders support the construction of coherent life narratives. The author proposes the term of "narrative accompaniment" to refer to the life narrative reelaboration processes of the personal history in non clinical contexts.
\end{abstract}

KEY WORDS Personal Narratives; Schizophrenia; Rehabilitation; Life Change Events; Community Mental Health Services. 


\section{INTRODUCCIÓN}

Las narrativas son un instrumento esencial mediante el cual construimos nuestras identidades (1-6). Según algunos autores una narrativa sobre sí mismo coherente y compartible en sociedad es una condición imprescindible para la completa integración social $(7,8)$. Podríamos decir que una narrativa sobre sí mismo es como una tarjeta de presentación en sociedad. Entendemos la identidad como un fenómeno empírico fundado en las acciones comunicativas de un sujeto enfrentado a la tarea de dar cuenta de su vida en situaciones específicas. Las situaciones a las cuales nos referimos pueden estar intensamente estructuradas (una exploración clínica) o formar parte de la vida cotidiana (por ejemplo, una conversación con un amigo en la barra de un bar). En cualquier caso, una narración es una acción muy compleja que ejecutamos en momentos de crisis o dentro de contextos clínicos. Una narración autobiográfica implica decidir qué acontecimientos de la vida son relevantes e imprescindibles, con qué calidad afectiva se deben presentar, cuántos detalles íntimos o problemáticos se deben incluir, y como en toda interacción social, cómo corresponder a las expectativas del interlocutor en la tarea narrativa. Por todo ello, no es extraño que en situaciones cotidianas no estemos acostumbrados a realizar estas tareas narrativas y que rememorar todas las experiencias vitales disponibles, seleccionarlas y llevarlas a la estructura de una narración en una situación social concreta requiera unos recursos cognitivos considerables.

¿Cuáles son las propiedades que logran que las narrativas se conviertan en un instrumento tan poderoso para la construcción de la identidad? Antes de proseguir y sin intención de ser exhaustivos apuntamos cuatro características esenciales de las narrativas:

1. Las narrativas pueden ser definidas como instrumentos cognitivos de naturaleza semiótica y por lo tanto cultural. Bruner (1) denomina a las narrativas como una forma de pensamiento, enfrentándola al "pensamiento paradigmático". Podríamos decir que en la narración, al contrario que, por ejemplo, en el lenguaje matemático, predomina la realidad psíquica. La existencia de un doble plano narrativo, el de la acción y el de la subjetividad de los personajes, es esencial en cualquier relato. Las narraciones muestran dos dimensiones; una dimensión objetiva, si queremos descriptiva y otra subjetiva. Esta última pertenece al plano psicológico de la conciencia. Podríamos hablar de una dimensión dual en las narrativas (1). Mediante una narración expresamos o descubrimos proyectos, las consecuencias de una acción, pensamientos ocultos (a veces contradictorios con una acción). Por lo tanto el lector o el oyente de una historia es ayudado a ingresar en las conciencias de los personajes y comprender las contradicciones de éstos; de esta manera aparece el verdadero drama humano. Además, gracias al carácter secuencial de las narrativas se facilitan la organización de las experiencias de vida y se posibilita la experiencia del tiempo.

2. Desde una perspectiva psicológica más centrada en el contexto social $(4,5)$ podríamos definir las narrativas como acciones lingüísticas construidas en interacciones sociales que pueden ser accesibles mediante métodos observacionales como fenómenos empíricos. El carácter social de las narrativas se observa con meridiana claridad cuando descubrimos en éstas modelos o formatos establecidos que nos ayudan a construir de forma aceptable nuestros relatos. Estas formas narrativas se han denominado de diversas maneras: géneros discursivos (9), discursos públicos (10) o en alemán original "Kulturell vorgeprägte Muster" (modelos culturales preestablecidos) (5). Géneros literarios, macronarraciones como historias nacionales o mitos comunitarios, o por ejemplo, en los últimos años, géneros conversacionales provenientes de los medios de comunicación de masas pueden funcionar como plantillas para construir nuestras historias.

3. La orientación social de todas las narrativas nos permite descubrir una de las funciones más interesantes de éstas para el trabajo en rehabilitación psicosocial. Todas las narrativas, especialmente las autobiográficas, presentan un componente evaluativo por el cual la audiencia se convierte en una instancia de 
aceptación o de rechazo (3). En este sentido podemos considerar una narración vital como una acción con una meta muy concreta: dar cuenta de la situación actual de un sujeto en el mundo (1). En otras palabras, justificar o hacer comprensible, comunicable, en un determinado marco sociocultural una determinada experiencia vital. De lo anterior se deduce que las narraciones vitales siempre permanecen en el plano de lo ético o de lo moral, como afirmó Ricoeur (11).

4. Debido a que las narraciones requieren de acciones, en las narraciones vitales se puede estudiar la capacidad de agencia de la cual disfruta el narrador en distintos aspectos de su narración. Es decir, si el narrador se describe como una persona con capacidad para influir en el mundo que le rodea y como centro de los acontecimientos que acontecen en la narración o simplemente se muestra como un ser sin ninguna capacidad de decisión ante distintos poderes ajenos a su persona. Además de ello, la construcción de una narración vital requiere motivación y de una actitud activa por parte del narrador. Por sí sola, la petición a una persona de dar cuenta de su situación vital la impulsa a situarse como un agente activo, a pesar de que se puedan encontrar en la narración vital pocos ejemplos de agentividad (5).

La esquizofrenia ha sido considerada desde los orígenes del concepto como una patología que reduce dramáticamente o imposibilita la capacidad de los pacientes de poseer un sentido coherente de sí mismo (12). Así, los pacientes serían incapaces de narrar de forma aceptable sus vidas y problemas. Los factores que han sido relacionados con la dificultad para la construcción de una historia de sí mismo coherente han sido numerosos: un rol de enfermo dominante y restrictivo, pérdida del reconocimiento de los déficits que se padece, intensidad de las emociones, autoestima amenazada, la existencia de evidentes déficits cognitivos y de síntomas negativos asociados a procesos orgánicos y, por último, disfuncionalidad de las conexiones lógicas. Son los déficits en las habilidades para pensar sobre los propios pensamientos, aquello que denominamos metacognición, lo que parece que se encuentra directamente implicado en las disfunciones de las narrativas de enfermos esquizofrénicos (13). Además, Holma y Aaltonen (7) nos alertan ante la presencia habitual en las experiencias de los pacientes esquizofrénicos de lo que estos autores Ilaman "subjugating stories", las cuales pueden destruir cualquier actividad de elaboración narrativa. En otras palabras, algunas experiencias narradas fragmentariamente pueden presentar propiedades de carácter traumático; por ejemplo: muerte de familiares, maltratos, abusos o acciones delictivas. Además, algunos autores han comenzado a estudiar la relación entre las experiencias traumáticas y los síntomas en la esquizofrenia, especialmente los síntomas positivos (14). El objetivo de este trabajo, no es analizar la relación entre la sintomatología y la etiología de las psicosis con las experiencias traumáticas de los pacientes y su articulación narrativa, sino más bien hacer énfasis en las necesidades sociales comunes que comparten enfermos y no enfermos. Nos referimos a la necesidad social de disfrutar de una historia de vida compartible en comunidad. Sin embargo, es indudable que el padecimiento de una psicosis implica una transformación radical de las experiencias de vida de las personas que las padecen hasta el punto que podemos decir que la misma psicosis es una experiencia traumática. Por lo tanto, en la discusión se expondrá muy brevemente desde qué ángulos teóricos se ha abordado la relación entre psicosis y trauma.

En la última década algunos investigadores han señalado que los pacientes no pierden nunca, o solo en momentos de crisis, su capacidad de explicar en un contexto social sus problemas, esperanzas y sentimientos (15). Desde el punto de vista de estas contribuciones el estudio de las experiencias subjetivas de los pacientes esquizofrénicos se convierte en un ámbito de investigación muy útil para los profesionales de salud mental. En esta misma línea se ha afirmado la necesidad de acercarse a las historias personales de carácter traumático y de integrar las dimensiones aisladas de la identidad dentro de una historia sobre sí mismo coherente. Conseguir el diálogo entre las distintas dimensiones del yo (posicionamientos) es uno de los objetivos fundamentales del proceso de adquisición de narrativas de vida coherentes $(16,17)$. 
Algunas contribuciones en los últimos años han señalado que los recursos psicosociales en salud mental deben ayudar a los usuarios a construir narraciones comunicables y coherentes sobre sus experiencias $(7,15)$. En este sentido, los hogares protegidos son recursos privilegiados para esta función. Pero, ¿cómo se puede acompañar a los pacientes en contextos rehabilitadores en su acercamiento a las historias de carácter traumático? ¿Cómo se puede ayudar a los pacientes a reelaborar sus historias $y$ transformarlas en narraciones coherentes $y$ compartibles? Hasta este momento se han descrito algunas intervenciones de carácter narrativo en distintos centros sociales y sanitarios de salud mental. Por ejemplo, podemos señalar el proyecto que se está Ilevando a cabo en estos momentos en la ciudad de Florencia en la Casa Famiglia "Meoste" di Bagno a Ripoli por los servicios sanitarios y el departamento de psicología de la Universidad de Florencia en base a una investigación anterior sobre la autobiografía como herramienta de construcción del self (18). En Río de Janeiro, Octavio Domont de Serpa (19) ha diseñado programas de formación de profesionales de salud mental incluyendo las experiencias subjetivas de los pacientes de un hospital de día, contando con la colaboración activa de los pacientes en los citados programas y considerando esta participación como la base para un nuevo marco terapéutico. También en América del Sur y en el contexto de las últimas transformaciones del sistema de gestión de salud mental, Palombini (20) y Lancetti (21) analizan cómo los nuevos escenarios terapéuticos desbordan los contextos clínicos tradicionales para introducirse dentro de la ciudad e introducen el concepto de acompañamiento terapéutico como un nuevo marco de intervención.

Probablemente el hecho de que los procesos de elaboración narrativos se encuentren ligados a contextos exclusivamente clínicos haya impedido que los diseños de intervenciones narrativas en recursos de carácter social para enfermos mentales hayan sido más frecuentes. Sin embargo, como veremos más adelante, estos recursos presentan importantes ventajas para estos procesos.

\section{CONTEXTO INSTITUCIONAL}

Los hechos que se describirán en el siguiente apartado le acontecieron al autor del presente artículo cuando trabajaba en una ciudad andaluza como monitor en una "casa hogar" perteneciente a la Fundación Andaluza para la Integración Social del Enfermo Mental (FAISEM). El autor elaboró un pequeño diario de carácter etnográfico el cual fue utilizado para la creación de este artículo. En primer lugar se describe brevemente el contexto institucional donde acontecieron los hechos. Posteriormente se apuntan algunos datos sobre el paciente, obviando sus datos personales y lugar de residencia para defender su privacidad y se describen los hechos a los que aludimos.

Las "casas hogares" son definidas como recursos sociales y están diseñadas para personas que padecen trastornos mentales graves. Estos alojamientos se encuentran en lugares muy céntricos. Es decir, las "casas hogares" son construidas en lugares accesibles, cerca de los recursos comunitarios normalizados. Las "casas hogares" son alojamientos de puertas abiertas con supervisión profesional las 24 horas del día. En estos alojamientos habitan de 16 a 20 usuarios, todos pacientes con trastornos mentales graves. Los aproximadamente 14 profesionales que trabajan en cada "casa hogar" son considerados monitores, es decir, si bien algunos cuentan con formación clínica o social, en cualquier caso su función es de supervisión, cuidado y apoyo emocional. El $89 \%$ de los pacientes que residen en las "casas hogares" de la región de Andalucía padecen esquizofrenia, trastorno esquizotípico o trastornos de ideas delirantes. El resto de los pacientes están diagnosticados de trastornos afectivos graves. El 33\% proviene de su casa, el 27,2\% de alguna institución sanitaria, el 19,2\% de los antiguos hospitales psiquiátricos y el $11,5 \%$ procede de los servicios sociales. El 9,1\% restante de los residentes en las "casas hogares" provienen de la calle o de otros lugares sin especificar. Del total de residentes reciben tratamiento farmacológico el $98,2 \%$, mientras que los que disfrutan de tratamiento psicosocial son solo el 38,8\%.

Las "casas hogares" muestran características de lo que se ha llamado "supportive housing 
model" (22). Por ejemplo, las "casas hogares" son escenarios en los cuales las rutinas diarias de los residentes son supervisadas por el equipo de profesionales. También los residentes comparten un número importante de áreas comunes. Por lo tanto podemos decir -aunque existan espacios privados para los residentes promovidos por la institución- que este modelo residencial implica la formación de un "group of life". Sin embargo, también podemos encontrar características que corresponden a un "supported model". Por ejemplo, existe una clara distinción entre el programa residencial y el tratamiento clínico o rehabilitador y como hemos dicho antes, se promueven espacios privados para los residentes. Dentro del contexto de las "casas hogares", los monitores tienen la misión principal de asegurar los cuidados básicos de los pacientes, supervisar su atención sociosanitaria y proveerles apoyo emocional. Exceptuando algunos objetivos básicos en habilidades sociales o autocuidados, los monitores no son responsables de ningún programa de intervención sistemático de carácter social o psicoeducativo. La intervención profesional de carácter clínico y psicosocial es responsabilidad exclusiva de los servicios sociosanitarios públicos. Sin embargo, resulta inevitable que los monitores intervengan habitualmente en escenarios como crisis psicóticas, conflictos grupales y problemas emocionales graves. A pesar de las reuniones de coordinación en cada "casa hogar" y de la progresiva profesionalización de los monitores en los últimos años, sus intervenciones están fuertemente influenciadas por su bagaje profesional, educativo, y cómo no, personal.

Mientras Jorge residía en esta institución acontecieron los hechos que se relatan en este artículo. El paciente tenía aproximadamente 30 años, padecía una esquizofrenia paranoide y sufría habitualmente alucinaciones auditivas. Llevaba viviendo en el hogar protegido varios años. Presentaba en ocasiones una conducta disruptiva y graves problemas para comunicarse debido a su discurso incoherente. Jorge estaba siendo tratado con antipsicóticos atípicos y acudía con regularidad a las revisiones clínicas normalizadas. Jorge procedía de una familia multiproblemática. Los informes sobre el paciente indicaban un entorno familiar problemático con sospechas de maltratos y abusos. La relación del paciente con su madre era positiva y ésta lo visitaba periódicamente con una buena respuesta del paciente.

\section{DESCRIPCIÓN DEL CASO}

Los episodios que se describen comenzaron a partir de una demanda muy concreta de Jorge. El paciente solicitó que se le acompañara al cementerio a visitar la lápida de su padre. Éste había fallecido un par de años atrás. La directora del centro donde residía el paciente le asignó a un monitor, con el acuerdo de Jorge, la tarea de acompañarlo al cementerio. A mediados de noviembre del año 2004 se realizó la primera visita al cementerio. La lápida del padre del paciente se situaba al final del cementerio, en el punto más lejano desde la entrada principal. Durante el camino que llevaba a la lápida del padre, que duró aproximadamente 20 minutos, Jorge comenzó espontáneamente a narrar escenas inconexas de su vida con su padre. Por ejemplo, los días de vacaciones en la playa o la celebración de algún cumpleaños. Estos relatos parecían ser producto de alguna ideación delusiva puesto que el paciente intercalaba escenas de distintos acontecimientos sin ningún tipo de orden temporal o espacial. Tras descubrir el contenido autobiográfico de los relatos, y con el objetivo de ayudar a Jorge a estructurar dichos relatos, se promovió la aparición de marcadores espaciales, temporales y de detalles mediante preguntas concretas. El tono emocional de estos relatos fragmentados era muy intenso, entre la nostalgia y la culpabilidad. En todo momento el paciente se mostraba muy unido emocionalmente a su padre. Al Ilegar a la zona donde se encontraba la lápida de su padre todavía tuvimos que dedicar más de diez minutos a buscarla. Jorge participó activamente en la búsqueda mientras proseguía relatando algunos sucesos. En más de una ocasión Jorge tomó la iniciativa en la búsqueda. Al llegar a la lápida Jorge se arrodilló, se persignó y dijo: "ipadre, nunca dejes de querer a tu hijo!". El paciente volvió a la residencia con normalidad y pasó un día sosegado.

Aproximadamente 20 días después el paciente demandó una nueva visita. En este caso 
a su antigua escuela de educación básica. El acompañamiento se asigna al mismo monitor por decisión directa del paciente. Ambos recorrieron las instalaciones del colegio. Visitaron aulas, patios de juegos y lugares de alrededor. Jorge se tomó un café en una cafetería que habitualmente visitaba en la época en la que estudiaba en el colegio. Reconoció lugares de los alrededores y a algunos profesores, probablemente de forma errónea. A pesar de ello, se le preguntó a Jorge por el carácter de estos profesores y su relación con ellos. Jorge contó historias muy pequeñas y simples, aunque, en esta ocasión, con una mínima coherencia temporal y espacial. En algunas de estas pequeñas historias el paciente aparece como protagonista y agente activo. Por ejemplo, mientras paseaba por las instalaciones deportivas narró un partido de fútbol que jugó con sus compañeros, en el que él fue portero y en el que tuvo una buena actuación. Las historias que contaba Jorge con relación a su vida en la escuela eran más controladas por éste que las que relataba respecto a su padre, las cuales presentaban una fuerte activación emocional que desestructuraba las narraciones. A la vuelta se le pregunta al paciente si su padre lo acompañaba al colegio. Jorge respondió muy lacónicamente: "a veces". El paciente volvió a la "casa hogar" con normalidad.

A mediados de febrero del 2005 Jorge vuelve a pedir una visita al cementerio. Las narraciones del paciente vuelven a mostrar una gran incoherencia, aunque en esta ocasión el tono emocional es más intenso. Las referencias del paciente a su padre son siempre muy positivas. Esta activación emocional no impide a Jorge mantener en todo momento un buen contacto comunicativo con el monitor. Ante la lápida se vuelve a arrodillar, rompe a llorar y dice que se "cortará las venas para estar con su padre". Cuando regresa a la "casa hogar" tiene un conflicto con un usuario de la residencia.

A principios de abril, aproximadamente seis meses después de la primera visita, el monitor vuelve a acompañarlo al colegio. Jorge prosigue con sus relatos sobre sus experiencias en el colegio. El monitor pregunta al paciente de forma que se conecte su posición como "yo-colegial" y "yo-hijo". Por ejemplo: "¿Te ayudaba tu padre con las tareas del colegio?". Poco a poco un tema va centrando todas sus narraciones: la relación de
Jorge con sus padres y hermanos. Habla mucho de su padre y se activa emocionalmente. Mientras que las referencias a su padre continuaban siendo positivas, por ejemplo cómo su padre iba a recogerlo a las puertas del colegio, en un plano conversacional (tono, intensidad de la voz, interrupciones) y no verbal (rigidez facial, inquietud motora), las señales eran de evidente incomodidad, e incluso, de violencia. En el camino de vuelta a la "casa hogar" el monitor intenta moderar la activación emocional de Jorge desviando la atención del paciente hacia otras temáticas pero la activación fisiológica de Jorge no deja de ascender. Bruscamente, poco después de volver a reafirmar su afecto por su padre, Jorge relata episodios de maltrato y abusos por parte de su padre y rompe a llorar. Al entrar en el taxi Jorge toma la palabra y pregunta al conductor: "¿Su padre también le pegaba con una correa?". Se informó a la dirección del centro y a los responsables sanitarios del paciente de estas experiencias. Tras estos episodios Jorge no volvió a solicitar nuevas visitas. No consta que volviera a hacer referencia a su padre como maltratador. Más allá del comportamiento sosegado, que en ocasiones podría denominarse triste, y de una disminución de conductas disruptivas, como gritos y soliloquios, en los siguientes días no se dispuso de una evaluación rigurosa que mostrara alguna mejora terapéutica tras sus cuatro visitas, dos al cementerio y dos a su antiguo colegio.

\section{DISCUSIÓN}

Podemos sugerir que Jorge con sus demandas de visita a su antiguo colegio y a la lápida de su padre nos estaba pidiendo ayuda para integrar ciertos aspectos de su historia personal que habían quedado desconectados. También podemos apuntar que el contacto directo bajo supervisión con escenarios significativos de la vida de Jorge y las interacciones con el monitor facilitaron la recuperación de experiencias y supusieron un primer paso para la integración coherente de éstas en una narración compartible. La visita supervisada a lugares significativos en el momento adecuado puede ser una estrategia interesante para activar la memoria y elaborar 
experiencias vitales que bloquearon las narrativas de aquellas personas que vivieron sucesos de carácter traumático. Desde esta perspectiva, reelaborar las historias personales es una de las dimensiones fundamentales del proceso de rehabilitación en personas que sufren trastornos mentales graves (23).

En los últimos años las historias traumáticas se han correlacionado con muchos de los síntomas que padecía Jorge, como por ejemplo con alucinaciones auditivas (14). En ocasiones, en el fondo de los discursos fragmentarios e incoherentes de carácter delusivo o alucinatorio de muchos de nuestros pacientes permanecen escondidas historias de abandono, maltrato o abuso, las cuales podrían ser comunicables en algún grado e integradas en las historias de los pacientes.

Las relaciones entre las experiencias traumáticas y las psicosis son extremadamente complejas. Para un extenso análisis de la cuestión es posible acudir a la revisión de Saavedra (24) publicada en Internet. Resulta evidente que algunos de los síntomas positivos de la psicosis presentan una morfología similar a las experiencias padecidas por personas con trastornos posttraumáticos y que las narraciones de estas experiencias muestran las mismas deficiencias estructurales que los relatos de personas que han sufrido tortura o experimentado alguna catástrofe. Aquí, solo se resumirán brevemente las tres principales perspectivas de estudio de estas relaciones a partir de un gran número de investigaciones, todas éstas citadas en la revisión de Saavedra (24). Un grupo de investigadores $(14,25,26)$ relaciona causalmente y de forma directa las experiencias traumáticas con las psicosis. Éstos consideran a algunas psicosis como una respuesta que se encuentra en el mismo espectro que los trastornos por estrés postraumáticos. Estos autores justifican la asociación de ambas patologías a partir de la hiperactivación del funcionamiento del eje hipotalámico-pituitario-adrenal. Incluso han acuñado el término de "psicosis disociativa postraumática". Otro grupo de investigadores $(27,28)$ consideran que las experiencias traumáticas provocan importantes distorsiones cognitivas y perceptivas, esquemas cognitivos distorsionados sobre uno mismo y el mundo, además de problemas en la adquisición de habilidades sociales necesarias para el contacto personal. Los problemas cognitivos y relacionales causarían que estas personas fueran especialmente vulnerables a experiencias psicóticas subclínicas que posteriormente podrían convertirse en psicopatológicas. Por último, otras investigaciones $(29,30)$ han considerado la psicosis y los tratamientos sociosanitarios como causantes de experiencias traumáticas, tanto es así que es posible predecir en pacientes de psicosis el diagnóstico futuro de un trastorno por estrés postraumático asociado. Sea cual sea la perspectiva más acertada, y del grado de distorsión de la memoria que sufra en nuestro caso Jorge, es evidente que los pacientes con psicosis necesitan de una ayuda especial para elaborar sus recuerdos en narrativas coherentes que sean comunicables desde un marco psicosocial. Lo cual, por otra parte, es requisito indispensable para todas las personas, enfermas o no, si se quiere ser reconocido y poder intervenir activamente en sociedad. La ayuda a las personas con, por ejemplo en nuestro caso, esquizofrenia, por hacer más coherentes y comunicables sus historias de vida, tiene como objetivo aumentar las posibilidades de los pacientes de integrarse en la sociedad. La meta de lograr una terapia que ataque el núcleo de la enfermedad mental y que pueda lograr algún grado de "curación", aunque esté conectada con las propuestas aquí planteadas, quedaría fuera del alcance de las intervenciones que se proponen en este artículo.

Más allá del contexto clínico ortodoxo de la consulta psicológica, proponemos la vida diaria en los nuevos centros sociales -por ejemplo la residencia donde vivía Jorge- como escenarios privilegiados para ayudar a los pacientes a reelaborar sus historias $(7,23,31)$. Además, estas narrativas pueden convertirse en instrumentos de evaluación del proceso de rehabilitación (32-34). Estos escenarios, por ejemplo, las "casas hogares", se encuentran muy alejados de los contextos clínicos y los profesionales que trabajan en estos centros, en muchos casos, conviven diariamente con los pacientes en un entorno muy cercano al familiar. En este contexto no se puede evitar que los pacientes nos cuenten historias personales. Muchas de éstas se encuentran fragmentadas, presentan una gran activación emocional y muestran contenidos de violencia, abandono o frustración. Estos alojamientos protegidos proveen a los pacientes de un importante apoyo emocional 
y aseguran las rutinas diarias relacionadas con las actividades básicas de la vida (alimentación, autocuidados, regulación del sueño) (31). Algunos autores han hecho énfasis en la importancia de la estabilidad y calidad de estas rutinas como el marco esencial del proceso de recuperación (32). Por lo tanto, y de acuerdo con otras aportaciones citadas $(20,21)$, estos escenarios son excelentes para promover, bajo rigurosa supervisión clínica, la reelaboración de experiencias que no han sido narradas coherentemente y que han bloqueado todo el flujo de la experiencia posterior.

En la experiencia que se ha informado hallamos tres aspectos que nos animan a pensar en estos hogares protegidos como espacios seguros para la elaboración de las narrativas de vida:

1. El reconocimiento por parte de la dirección del hogar protegido de la importancia de la petición del paciente y la disposición de la dirección de dicho hogar a financiar el desplazamiento del paciente.

2. La adjudicación de un monitor para su acompañamiento. Este hecho es importante teniendo en cuenta que los recursos humanos son limitados. Además se respetó la decisión de éste respecto a la identidad del monitor. Este último es un punto esencial, puesto que un acompañamiento indeseado obstruiría en gran parte el proceso de elaboración.

3. La iniciativa del monitor, el cual no se limitó a supervisar el comportamiento del paciente y a contener su activación emocional, sino que fue receptor pasivo de sus historias promoviendo su elaboración y estructuración.

Sin embargo, las excelentes posibilidades "terapéuticas" y "rehabilitadoras" de estos recursos todavía no son formalmente reconocidas en el contexto donde se ha desarrollado el caso que hemos analizado así como en otros muchos lugares. En los objetivos de estos hogares protegidos no aparecen metas que aborden estos aspectos y los profesionales que allí trabajan no son entrenados para ello. De este modo, los servicios sanitarios no consideran de forma clara a estos recursos, ni a los profesionales que allí trabajan, como agentes activos y decisivos en las mejoras terapéuticas de los pacientes. Por lo cual cualquier tipo de intervención de carácter psicosocial o terapéutica se ve limitada.

Los aspectos que se señalan a continuación -considerados esenciales en el proceso de acompañamiento narrativo- están referidos al trabajo con personas que padecen trastornos mentales graves en recursos de carácter social como lo son los hogares protegidos, aunque pueden generalizarse a otras poblaciones de personas con problemas de integración o con experiencias de carácter traumático (mujeres maltratadas, inmigrantes irregulares, refugiados de guerra, etc). Ya que, como hemos señalado, una narrativa de vida comunicable es un requisito imprescindible para la vida social de todos los seres humanos.

1. En el contexto de los hogares protegidos existe un compromiso personal entre las personas que padecen trastornos mentales graves y los profesionales. En algunos casos, cercano a la amistad, y siempre de un conocimiento mutuo significativo. Este hecho diferencia el escenario de los hogares protegidos del contexto clínico de la consulta psiquiátrica o de la intervención médica. Para acompañar a la elaboración de narrativas traumáticas es importante una relación excelente entre el profesional y el residente en el hogar protegido. En el episodio que hemos descrito Jorge selecciona al monitor por la confianza de la cual disfruta con él.

2. Es importante señalar que los pacientes deben siempre expresar su acuerdo en el inicio de los procesos de acercamiento a sus historias traumáticas. Forzar situaciones no buscadas por los pacientes puede provocar el surgimiento de nuevos síntomas o el agravamiento de los existentes. En nuestro caso, la demanda de Jorge es evidente y nos indicó que se encontraba preparado para el acercamiento a sus historias no narradas. Es necesario esperar señales que nos indiquen que las personas están preparadas para elaborar sus historias traumáticas y no tomar la iniciativa. Estas señales no son siempre tan claras como en el caso que hemos descrito. En ocasiones consisten en expresiones vagas de recuerdos, preguntas a los monitores sobre cuestiones familiares o afectivas o reacciones emocionales destacables en fechas significativas como Navidad o en cumpleaños. En cualquier caso, 
hay que asumir algunos riesgos cuando a partir de signos se decide explorar algunas experiencias que parecen desestructuradas.

3. Es esencial ser paciente, escuchar activamente y permitir el discurso incoherente o aparentemente delirante. No rechazarlo ni desvalorarlo. No empezar a intervenir inmediatamente realizando preguntas o pretendiendo estructurar el discurso. Es fundamental que atribuyamos significado a lo que no comprendemos del discurso de la persona con la cual hablemos, por absurdo que nos parezca.

4. Ayudar a estructurar lo que nos cuentan. No cansarnos de pedir referencias: dónde, cuándo, quién. Muchos relatos aparentemente sin sentido adquieren significado cuando pedimos información adicional a nuestro interlocutor para situar el escenario y los protagonistas de las historias. Muchas personas piensan que se comparte más información de la que realmente se conoce y ahorran referencias. Esto ocurre especialmente en las personas que padecen de esquizofrenia.

5. Ayudar a que las distintas dimensiones de la identidad tomen contacto. Cuando los intereses, los deseos o las necesidades de los distintos posicionamientos entran en diálogo podemos empezar a reconocer la complejidad de los vínculos humanos y comprender que una relación puede presentar aspectos positivos y negativos. No es absurdo amar a una persona que nos ha hecho daño. En el episodio que hemos descrito el monitor preguntó a Jorge con la intención de contactar el "yo-hijo", un posicionamiento muy problemático para Jorge, con el "yo-estudiante" que aparentemente no presentaba aspectos negativos. En el caso descrito, no es posible afirmar con seguridad que el contacto entre estos dos posicionamientos haya provocado el reconocimiento del padre como "maltratador" en Jorge; y en el caso que así fuera, cuáles fueron las causas concretas de este efecto positivo. Ello requeriría un análisis más profundo. En cualquier caso, cuanto más contacto entre los posiciona- mientos exista, más posibilidades hay de que surjan experiencias reprimidas y emociones que no han sido nombradas. En la experiencia que hemos expuesto con Jorge hemos visto cómo acercarse a escenarios significativos en la vida puede ayudarnos a activar la memoria y a elaborar distintas historias. Esto ayudó a que Jorge construyera una historia más compleja. Acudir a fotografías o canciones pueden ser recursos interesantes para trabajar las historias personales. De igual forma, participar en talleres creativos, en los cuales por medio de la pintura o cualquier otra actividad se pueda dar forma material a distintas emociones, es una actividad muy útil y gratificante para muchas personas.

6. Estar atento al lenguaje corporal. Ayudar a éste y al lenguaje verbal a que sean coherentes uno respecto al otro. Dar nombre a las emociones.

7. No buscar en todos los casos la descripción exhaustiva de la experiencia traumática. Existen muchas formas de integrar experiencias. Expresiones como "mi padre me hizo mucho daño y todavía no lo he superado" o "no tengo palabras para explicar cómo sufrí en aquel momento" pueden ser suficientes. En cualquier caso, es imprescindible respetar los tiempos y las necesidades de cada persona. Se pueden trabajar los sentimientos de culpa e impotencia sin centrarse en la descripción de la experiencia traumática.

8. Reconocer las situaciones de riesgo y saber volver atrás. En cualquier caso, es esencial mantener informada a la dirección del centro y a los profesionales de referencia de cualquier exploración de carácter narrativo.

A partir de todo lo expuesto podríamos denominar acompañamiento narrativo (AN) a todo tipo de ayuda profesional que conlleve la reelaboración de las narrativas de vida de los pacientes en un contexto de rehabilitación psicosocial. 


\section{REFERENCIAS BIBLIOGRÁFICAS}

1. Bruner J. Actos de significado. Madrid: Alianza Editorial; 1991.

2. Salvatore G, Dimaggio G, Semerari A. A model of narrative development: Implications for understanding psychopathology and guiding therapy. Psychology and Psychotherapy: Theory, Research and Practice. 2004;77(2):231-254.

3. Gergen KJ. Erzählung, moralische Identität und historisches Bewusstsein. Eine sozialkonstructionistische Darstellung. En: Straub J, editor. Identität und historishces Bewusstsein. Frankfurt: Suhrkamp, Swarthmore Collage; 1998.

4. Gergen KJ, Gergen MM. Narrative form and the construction of psychological science. En: Sarbin TR, editor. Narrative psychology. Nueva York: Praeger; 1986.

5. Lucius-Hoene G, Deppermann A. Rekonstruktion narrativer Identität. Ein Arbeitsbuch zur Analyse narrativer Interviews. Opladen: Leske and Budrich; 2002.

6. McAdams D. The development of a narrative identity. En: Buss DM, Cantor $\mathrm{N}$, editores. Personality psychology: recent, trends and emerging directions. Nueva York: Springer; 1989.

7. Holma J, Aaltonen J. The sense of agency and the search for narrative in acute psychosis. Contemporary Family Therapy. 1997;19(4):463477.

8. Lysaker PH, Wickett AM, Wilke N, Lysaker J. Narrative incoherence in schizophrenia: The absent agent-protagonist and the collapse of internal dialogue. American Journal Psychotherapy. 2003;57(2):153-164.

9. Bakhtín M. Speech genres and other late essays. Austin: University of Texas Press; 1987.

10. Gergen KJ. El yo saturado: dilemas de identidad en el mundo contemporáneo. Barcelona: Paidos Contextos; 1997.

11. Ricoeur P. Tiempo y narración. Madrid: Cristiandad; 1987.

12. Sass LA. Negative symptoms, schizophrenia, and the self. International Journal of Psychology and Psychological Therapy. 2003;3(2):153-180.

13. Lysaker $\mathrm{PH}$, Carcione A, Dimaggio G, Johannessen JK, Nicoló G, Procacci M, Semerari A.
Metacognition amidst narratives of self and illness in schizophrenia: associations with neurocognition, symptoms, insight and quality of life. Acta Psychiatrica Scandinavica. 2005; 112(1):64-71.

14. Read J, Godman L, Morrison AP, Ross R, Aderhold V. Childhood trauma, loss and stress. En: Read J, Mosher LR, Bentall RP. Models of Madness. Nueva York: ISPS Publications; 2005.

15. Roe D, Davidson L. Self and narrative in schizophrenia: time to author a new story. Medical Humanities. 2005;31(2):89-94.

16. Lysaker $\mathrm{PH}$, Lancaster RS, Lysaker JT. Narrative transformation as an outcome in the psychotherapy of schizophrenia. Psychology and Psychotherapy. 2003;76(3):285-299.

17. Lysaker PH, Lysaker J. Narrative structure in psychosis schizophrenia and disruptions in the dialogical self. Theory \& Psychology. 2002;12(2):207-220.

18. Smorti A, Risaliti F, Pananti B, Cipriano V. Autobiography as a tool for self construction: a study of patients with mental disorders. Journal of Nervous and Mental Disease. 2008;196(7):531537.

19. De Serpa Junior OD, Martín E, Ramos RC, Da Silva JF. Including subjectivity in the teaching of Psychopathology. Interface. 2007;11(22):207-222.

20. Lima Palombini A. Vertigens de uma psicanálise a céu aberto: a cidade. Contribuições do acompanhamento terapêutico à clínica na reforma psiquiátrica. [Tesis de doctorado en línea] Río de Janeiro: Universidade Estadual do Rio de Janeiro; 2007 [fecha de acceso 25 de febrero de 2009]. URL disponible en: http://www.lume.ufrgs.br/bitstream/handle/10183/10191/000596452.pdf? sequence $=1$

21. Lancetti A. Clínica peripatética. San Pablo: Politicas do Desejo; 2007.

22. Dorvil H, Morin P, Beaulieu A, Robert D. Housing as a social integration factor for people classified as mentally ill. Housing Studies. 2005; 20(3):497-519.

23. Sells DJ, Stanyner DA, Davidson L. Recovering the self in schizophrenia: An integrative review of qualitative studies. Psyquiatric Quarterly. 2004;75(1):87-97.

24. Saavedra J. Un espíritu negro me entró en la boca. Narrativas incoherentes de pacientes esquizofrénicos y narrativas de eventos traumáticos. 
[Resumen en línea] Conferencia presentada en el $9^{\circ}$ Congreso Virtual de Psiquiatría Interpsiquis 2008 [fecha de acceso 18 de diciembre de 2008]. URL disponible en: http://www.psiquiatria.com/ articulos/psicosis/33441/

25. Read J, Perry B, Moskowitz A, Connolly J. The contribution of early traumatic events to schizophrenia in some patients: a Traumagenic Neurodevelopmental model. Psychiatry. 2001;64(4):319-345.

26. Seedat S, Stein M, Oosthuizen P, Emsley R, et al. PTSD and psychosis: a look at epidemiology, phenomenology, and treatment. Journal of Nervous and Mental Disease. 2003;191(10):675-681.

27. Steel C, Fowler D, Holmes EA. Trauma-related intrusions and psychosis: an information processing account. Behavioural and Cognitive Psychotherapy. 2005;33(2):139-152.

28. Bak M, Krabbendam L, Janssen I, de Graaf R, Vollebergh W, van Os J. Early trauma may increase the risk for psychotic experiences by impacting on emotional response and perception of control. Acta Psychiatrica Scandinavica. 2005; 112(5):360-366.

29. Chisholm B, Freeman D, Cooke A. Identifying potential predictors of PTSD reactions to psychotic episodes. British Journal of Clinical Psychology. 2006;45(4):545-559.
30. Shaw K, McFarlane A, Bookless C, Air T. The aetiology of postpsychotic posttraumatic stress disorder following a psychotic episode. Journal of Traumatic Stress. 2002;15(1):39-47.

31. Saavedra J. Schizophrenia, Narrative and Change: Andalusian Care Homes as Novel Sociocultural Context. [Abstract en línea] Culture, Medicine and Psychiatry. 19 de febrero de 2009 [fecha de acceso 5 de marzo de 2009]. URL disponible en: http://www.ncbi.nlm.nih.gov/pubmed/19221869?dopt $=$ Abstract

32. Jenkins JH, Carpenter-Song E. The new paradigm of recovery from schizophrenia: cultural conundrums of improvement without cure. Culture, Medicine and Psychiatry. 2005;29(4): 379-413.

33. Ridgway P. Restorying Psychiatric disability: learning from first person recovery narratives. Psychiatric Rehabilitation Journal. 2001;24(4):335343.

34. Saavedra J. Changes in the life narratives of schizophrenic patients after staying in special boarding houses in Andalusia. [Resumen] Trabajo presentado en el 15th International Congress for the Psychotherapy of Schizophrenia and other Psychoses. Acta Psychiatrica Scandinavica. 2006;114(S431):S40.

\section{FORMA DE CITAR}

Saavedra Macías J. ¿Cómo acercarse a historias traumáticas en contextos de rehabilitación psicosocial? Un estudio de caso de un paciente de esquizofrenia paranoide. Salud Colectiva. 2009;5(2):225-235.

Recibido el 9 de diciembre de 2008

Versión final presentada el 10 de marzo de 2009

Aprobado el 25 de marzo de 2009 